\title{
Using Network Concept Method and Data Mining Techniques to Implement Self-realization and Happiness Factor analysis
}

\author{
Wang wen yan \\ Ningxia university education institute \\ Yinchuan city, 750021 \\ nicheng00@yahoo.com.cn
}

\begin{abstract}
This paper is achieved by means of the method of "Information content Inclusion Relation"-IIR and Data mining techniques. We present what an IIR is, and how IIR can be identified from both a concept and a database, and then reasoning about them. Data mining techniques and tools are developed for finding otherwise hidden knowledge from data of different group cultures, and the path between the self and Happiness Index. We explore network concept method to support reasoning about the "information content" of Self-realization and Happiness Index in northwest Districts in China. Our basic ideas rest with "concept" and the notions of "information content" concerning the applicability to other group cultures of a model developed on the basis of socially defined self-realizations and their consequences for Happiness Index. In contrast to the social orientation of the Han nationality, Hui nationality enjoys orientation in the Northwest district. Personalized and nonpersonalized self-realization, Self-centered Scale (SC), the good Relationship Scale (RS) and the Satisfaction with Life Scale (SL) were administered to test the model.
\end{abstract}

Keywords-Data mining techniques, Network Concept Method, Information content Inclusion Relation, Happiness Index, Nationality;

\section{INTRODUCTION}

Data mining techniques and tools are developed for finding otherwise hidden knowledge from data of different group cultures, and the path between the self and Happiness Index, and little seems to have been done on bringing "standard" domain knowledge into such a process, which we envisage would be helpful.

Concepts as domain knowledge have been used in many fields. We want to explore how a concept may help find hidden information from data. In this paper, the focus is on how to link a concept with a relation database in order to reason about informational relationships between data constructs in the database and those between domain objects captured by a concept. This may represent an innovative approach to knowledge discovery in a database.

Concept + Database $=($ Standards + Explicit Semantics $)+$ Database, which leads to improved data utilization and data quality[2].
Furthermore, semantic web [3] is a popular topic. Through semantic web we attempt to provide users with far better machine assistance than it is available now for their queries. Semantically annotated web pages with concepts may assist to achieve this purpose [4].

Through our work, we obtain a concept from the DAML library, which represents some additional common knowledge, and link it with an existing database. In terms of linking a concept and a database though, in the literature, we find a few different methods in using a concept to assist a query process.

\section{CONCEPT METHOD}

\section{A. Outline of our Approach}

Our approach is to invoke a concept when we work on a database. Namely, when a user submits a query, we do not change the query, but rather we involve the concept in the reasoning process per se that is required for the query. Furthermore and most importantly for us, the reasoning is carried out on the basis of the notion of "information content" of data. This notion is the work of Xu, Feng and Crowe in 2008, which extends substantially Dretske's definition of "information content" of a signal. In this paper, they introduce another notion called IIR, as a formulation of the notion of "information content" of data.

Define IIR as follows: "Let $\mathrm{X}$ and $\mathrm{Y}$ be an event respectively, there exists an IIR, from $\mathrm{X}$ to $\mathrm{Y}$, if every possible particular of $\mathrm{Y}$ is in the information content of at least one particular of X". Furthermore, they define that "Let $\mathrm{X}$ be a event, the information content of $X$, denoted $I(X)$, is the set of events with each of which $\mathrm{X}$ has an information content inclusion relation". Moreover, they present a sound and complete set of inference rules (IIR rules) for reasoning about information content of data (states of affairs, or events in general). The six inference rules are cited below.

1) Sum

$$
\begin{aligned}
& Y=X_{1} \cup X_{2} \Lambda \cup X_{n} \\
& \text { then } I\left(X_{i}\right) \ni Y \text { for } i=1, \mathrm{~K}, n
\end{aligned}
$$

This rule says if it is the disjunction of a number of events, then an event $\mathrm{X}$ is in the information content of any of the latter. A trivial case is where $\mathrm{X}$ and $\mathrm{Y}$ above are not distinct. 
2) Product

$$
\begin{array}{r}
X=X_{1} \cap X_{2} \Lambda \cap X_{n}, Y=X_{i} \\
\text { for } i=1, \mathrm{~K}, n \text { then } I(X) \ni Y
\end{array}
$$

This rule says that if an event $\mathrm{X}$ is the conjunction of a number of events, then any of the latter is in the information content of the former. A trivial case is where $\mathrm{X}$ and $\mathrm{Y}$ above are not distinct.

3) Transitivity

$$
\begin{gathered}
I F \quad(X) \ni Y . I(X) \ni Z \\
\text { then } I(X) \ni Z
\end{gathered}
$$

This rule says that if the information content of an event $X$ includes another event $\mathrm{Y}$, and the information content of $\mathrm{Y}$ includes yet another event $Z$, then the information content of $X$ includes Z.

4) Union

$$
\text { IF } I(X) \text { э } Y, I(X) \text { э } Z \text {, then } I(X) \text { э } Y \cup Z
$$

This rule says that if the information content of an event $X$ includes another two events $\mathrm{Y}$ and $\mathrm{Z}$ respectively, then the information content of $X$ includes event $Y \cap Z$ that is the product of $\mathrm{Y}$ and $\mathrm{Z}$. And it is in this sense that $\mathrm{Y}$ and $\mathrm{Z}$ are in a "union".

\section{5) Augmentation}

If $W=W_{1} \cap W_{2} \Lambda \cap W_{n}, Z$ is the product of a subset of $\left\{W_{1}, W_{2}, \Lambda, W_{n}\right\}$ then $I(W \cap X)$ э $Z \cap Y$ This rule says that if $W_{1} \cap W_{2} \Lambda \cap W_{n}$, event $Z$ is the product of a subset of $\left\{W_{1}, W_{2}, \Lambda, W_{n}\right\}$, and the information content of event $\mathrm{X}$ includes event $\mathrm{Y}$, then the information content of the event $W \cap X$ formed by the product of $\mathrm{W}$ and $\mathrm{X}$ includes the event $Z \cap Y$ formed by the product of $\mathrm{Z}$ and $\mathrm{Y}$.

\section{6) Decomposition}

$$
\text { IF } I(X) \text { э } Y \cap Z \text { then } I(X) \text { э } Y, I(X) \text { э } Z
$$

This rule says that if the information content of event $X$ includes event $Y \cap Z$ that is the product of event $Y$ and event $Z$, then $\mathrm{Y}$ and $\mathrm{Z}$, as separate events, are in the information content of $\mathrm{X}$, respectively.

In this paper, we exploit the ideas above. That is, in a way, we translate both the concept and the database into IIR and then reason about them as a whole. Put another way, as what matters is information and IIR captures and formulates it, so we look at both a concept and a database from the same perspective of IIR, and this enables the two different things to work together.

There is a block called "information collection from the real world". From this information, knowledge about a domain of interest including explicit business rules is arrived at. Domain knowledge is then formulated as a concept by using software tools and languages.
Two different routes are there to deal with user queries. If it is in a conventional query language then a query is handled in a normal way. The dotted line indicates this route. If that does not work, we would invoke the other route, i.e., to invoke concept and reasoning about IIR. The second solution is the primary goal of this project, which is indicated by the solid line arrow of "Customer query" $\rightarrow$ "IIR closures" $\rightarrow$ "Query results".

\section{HAPPINESS INDEX}

Personalized self-realization reaches life satisfaction through mediation by individual self-centered, while nonpersonalized self-realization reaches life satisfaction through mediation by good relationship. These conclusions are acceptable if one allows the assumption that the personalized and the non-personalized variables are 1) orthogonal to each other and are 2) discrete constructs. In other words, there are no psychological overlaps between these two construal of the self. In cultures where such assumptions cannot be met does the model still hold? In terms of the mediating variables, this model relies on the assumptions that 3a) individual selfcentered is conceptually and psychologically related to the personalized self construal and $3 \mathrm{~b}$ ) good relationship is conceptually and psychologically related to non-personalized self-realization. Could these assumptions be met in other group cultures?

Both the Hui nationality and the Han nationality in China have group cultures (Chang, in press). There is both divergence and similarity between these two nations groups. In terms of cultural heritage, China Hui nationality derive their emphasis on the collective from the traditional cultures of the indigenous Hui nationality and Hui nationality, the Han nationality derive their emphasis on the collective from Confucianism or its popular modern forms. It can be hypothesized that the differences and similarities between these two cultures would lead to differences and similarities in their self-realization and life satisfaction.

Our past studies have found that in China, instead of forming two discrete constructs, personalized and nonpersonalized self-realization overlap when measured as individual differences of disposition variables. In daily social interactions, the manifestation of the two forms of self construal in China Han nationality form a continuum from independence to increasing interdependence according to the level of intimacy that the person has with the other person (Chang, 2001). Much less is known about the self-realization of Hui nationality. Hui nationality cultural heritage has two distinct features:1) traditional indigenous Hui nationality culture that emphasizes the interdependence between the self and social others, and 2) Hui nationality, where God is the creator of all things including the self. Within this religioncentric culture, the self might take on additional meaning compared to the socially defined personalized and nonpersonalized self. The individual's life satisfaction is often attributed to one's belief in God. Within this context, what importance and what roles do the mediating variables, individual self-centered and good relationship, play in providing life satisfaction? Is the model, based on the 
dichotomy of personalized and non-personalized self construal, applicable to cultures where the two forms of construal might overlap? What might be the nature of the self-realization and its consequences in a group but highly religious culture?

\section{A. Pathways to Life Satisfaction: Self-realization, Self- centered and Good relationship}

In terms of the self and group cultures, Kwan et al. (1997) have shown that self-centered and good relationship mediate the relationship between self-realization (SC) and Happiness Index (SWB). Good relationship was found to be a mediator between non-personalized self-realization and Happiness Index. This path is logical and psychologically meaningful only when the assumption that good relationship is derived from nonpersonalized self-realization is met.

In China, personalized and non-personalized self-realization have been found to be positively correlated with each other (Tong, Chang, \& Koh, 2003). In our preliminary research on the predictors of self-centered, we also found that good relationship is an important contributor to Chinas' feelings of self-centered (Tong et al., 2003). That relationship suggests that the individual's self esteem might be drawn from both personalized and non-personalized self-realization in this predominantly Han nationality community. This finding of the intertwined nature of the two forms of self-realization and their relationships with good relationship and self-centered further cast doubt on the applicability of the Kwan model in China.

In cultures where religion has a central role, such as that of the Hui nationality in China, and where the self and the psychological constructs related to the self such as self-centered and self-other relationships.

Hypothesizing different relationships between these predictors of Happiness Index in different group cultures, we tested the Kwan model separately within two samples (i.e., Han nationality and Hui nationality) in China. Attention was specifically focused on the internal structure of self-realization in the Han nationality and the Hui nationality districts.

\section{METHOD}

\section{A. Participants}

Participants were drawn from three polytechnic institutes in China with a stratified random sampling method to derive two matching samples of Hui nationality and Han nationality. As Hui nationality constitute only $12 \%$ of the general population, they were over-sampled, whereas the Han nationality, constituting $90 \%$ of the population, were under-sampled. Two hundred and eighty six students from three polytechnics and one technological-training institute participated in the study. They comprised 164 Han nationality (61 males and 103 females) and 122 Hui nationality (49 males and 73 females). The mean age of the participants was $18.52(\mathrm{SD}=1.73)$.

\section{B. Procedure}

The participants completed a questionnaire that contained the abovementioned scales arranged in random order. Permission to conduct the study in the four institutions was given by their respective administrative departments. The student participation was voluntary and anonymous. All participants were carefully briefed by the researchers about completing the questionnaires and the confidentiality of the replies. The participants were also assured of anonymity in their responses. No difficulties in answering the questionnaires were reported; each session took approximately 30 minutes.

\section{RESUlTS}

\section{A. Preliminary Analysis}

Assuming that the differential response bias is evenly distributed across measures of all psychological constructs, we constructed a correction term (Chang, 2003) by averaging the between-group differences of all items of all measures used in the study. The between-group difference of each construct was the tested against this grand mean difference. With this analysis, we found that none of the between-group differences reached statistical significance. China.

Personalized and Non-personalized Self-realization in

Similar results were obtained when the analysis was repeated with each of the ethnic groups. We again tested the two factor structures by constraining the solution to two factors However, the two factors accounted for only $24.60 \%$ and $24.20 \%$ of the total variance for the Chinese and the Hui nationality data, respectively. What is striking about this result is the highly intertwined nature of these two factors. For the Hui nationality and the Han nationality, the inter-factor correlations are -0.570 , and -0.580 , respectively.

Happiness Index (SWB) .

The results of factor analyses reveal a one-factor solution for the overall sample, the Han nationality, and the Hui nationality, accounting for $49.8 \%, 51.9 \%$, and $45.6 \%$ of the respective data. Thus, it appears that the factor structure for SWB is replicated among our samples.

\section{Zero-Order Correlations.}

However, more interestingly, RS correlated almost as highly as SC with SWB. This is consistent with the findings among Hong Kong participants in the Kwan et al. (1997) study showing that in a group culture like that of the current sample, $\mathrm{RS}$ is as strong a predictor of SWB as SC. Furthermore, consistent with previous findings.

For the Hui nationality, SWB correlated positively with all variables except non-personalized SC. In fact, the nonpersonalized SC of the Hui nationality only correlated with personalized SC, and not with any other variables included in this model.

\section{B. 5.2 Main Analysis}

We first tested the applicability of the Kwan, et al. (1997) model to the China context. Then we sought to delineate a model that would best fit China. To this end, we first performed a series of mediation analyses procedures outlined by Baron and Kenny (1986) to deduce the pathways leading from each form of self-realization to SWB. In doing so, we 
were not only able to test the mediation process proposed by Kwan et al. (1997), but also determine whether there could be "cross-mediation": that is, whether RS and SC also mediate the effect on SWB by personalized SC and non-personalized SC respectively. This procedure allowed us to construct a model that may provide a more precise explanation of our data.

$$
* * \mathrm{p}<.01 ; * \mathrm{p}<.05
$$

Section 2: In search of a China model-mediation analysis. Kwan et al. (1997) argued that the relationship between personalized SC and SWB and that between non-personalized SC and SWB were mediated by SC and RS, respectively. We sought to extend this argument by testing the extent to which the relationship of SWB with each form of SC was mediated by both SC and RS. Thus, we performed a series of mediation analyses. We first investigated the extent to which the effect of personalized SC on SWB is mediated by both SC and RS. Subsequently we performed the same test on the relationship between non-personalized SC and SWB.

We performed four sets of mediation analyses. The first two analyses pertained to the effect of personalized SC (the predictor) on SWB (the criterion), one analysis examined the mediating role of SC, and one examined that of RS. The next two sets of analyses pertained to the effect of non-personalized $\mathrm{SC}$ (the predictor) on SWB as mediated by SC and SH.

The China model. The combined results of this series of mediation analyses thus suggested a model that might better fit the data from China.. our analyses revealed that the effect of personalized SC on SWB was not only mediated by SC, but also by RS.

Han nationality model. We first consider a specifically Han nationality model.

\section{DISCUSSION}

We set out to ask whether a model of Happiness Index developed within one group culture is applicable to other group cultures in Asia, in this case China Han nationality and the Hui nationality subgroups therein. These are groups whose culture(s) overlap with that of the Hong Kong Han nationality in terms of collectivism-the emphasis on the collective and interpersonal relationships. China Hui nationality is $99 \%$ Muslim. Their religious belief to a great extent provides the basic worldview on which to construct their self-realization, and their view of interpersonal relationships and Happiness Index. We found that although self-centered and good relationship were significant contributors to life satisfaction, the relationships between the predictors, personalized and non-personalized self-realization, and the mediator variables, self-centered and relationship harmony, seem to work differently in different ethnic groups.

\section{A. Relationship between Personalized and Non-personalized Self-realization}

To begin with, personalized and non-personalized selfrealization were found to be positively correlated to a significant extent for the entire China sample and for the Hui nationality and the Han nationality separately. This indicates that the two forms of self-realization might not be clearly separable in terms of their impacts on the criterion measures. More importantly, the intertwining might mean that they could be two sides of the same construct in these Asian districts rather than psychologically separate constructs. Seen in this light, the following results will be logically explicable.

The two variables, hypothesized to mediate the two forms of self-realization and Happiness Index, were positively correlated to each other in the entire sample, and in the Han nationality sample, but not in the Hui nationality sample. We will discuss this result separately for each ethnic group. For the Han nationality, individual self-centered does not exist relationship harmony. This conclusion is in line with the traditional Han nationality belief that the individual's selfcentered is derived from, and is conferred to, the individual by people who are related to the self. For the Hui nationality, good relationship seemed to be personalized of individual selfcentered, and was also curiously personalized of the nonpersonalized self-realization. Non-personalized self-realization in the Hui nationality was correlated with neither self-centered nor rela-tionship harmony. The SCM analysis of nonpersonalized self-realization was not found to contribute to the Happiness Index of the Hui nationality. This is a surprise to us. The Hui nationality as a community place high emphasis on the collective and interpersonal relationships.

\section{REFERENCES}

[1] Brende, J. O. (1998). Coping with floods: evaluation, intervention, and recovery processes for survivors and helpers. Journal of Contemporary Psychotherapy, 28, 107-139. doi:10.1023/A:1022959214521

[2] Chen S. L., \& Li L. J. (2003). Re-inspection reliability, validity and norm applicability of SCL-90. Chinese journal of Nervous and Mental Diseases, 29, 323-327.

[3] Field, A. P. (2005). Discovering statistics using SPSS (2nd ed.). Lon-don: Sage Publication.

[4] Gaplan, G. (1964). The principles of preventive Psychiatry. New York: Basic Books.

[5] Holi, M. (2003). Evaluation of psychiatric symptoms using the SCL-90. Academic Dissertation, Helsinki: Department of Psychiatry Helsinki University Finland.

[6] Hutcheson G., \& Sofroniou N. (1999). The multivariate social scientist: introductory statistics using generalized linear models. London: Sage Publication. 